\title{
An Autonomous Pesticide Sprayer Robot with a Color-based Vision System
}

\author{
Mona Tahmasebi ${ }^{\mathrm{a}, 1, *}$, Mohammad Gohari ${ }^{\mathrm{b}, 2}$, Alireza Emami ${ }^{\mathrm{b}, 3}$ \\ a Agricultural Engineering Research Department, Markazi Agricultural and Natural Resources Research and Education \\ Center, Agricultural Research, Education and Extension, Organization (AREEO), Arak, 3818385149, Iran \\ ${ }^{\mathrm{b}}$ Department of Solid Mechanics, Faculty of Mechanical Engineering, Arak University of Technology, Arak, \\ 3818146763, Iran \\ ${ }^{1}$ tahmasebi.mona@gmail.com; ${ }^{2}$ moh-gohari@arakut.ac.ir; ${ }^{3}$ alireza.e.aut@gmail.com \\ * Corresponding Author: Mona Tahmasebi
}

\section{ARTICLE INFO}

\section{Article history}

Received October 13, 2021

Revised January 02, 2022

Accepted February 18, 2022

\section{Keywords}

Wheeled robot;

Pesticides;

Color sensor;

Kinematics;

Dynamics

\begin{abstract}
The usage of robots has been due to cost reduction and increasing operation accuracy. Furthermore, employing robots in farming can decrease human tasks in hard and dangerous duties such as plowing, spraying pesticides, etc. Spraying chemicals is a common operation in agriculture crop protection. This operation is essential, but it can create some problems such as human and environmental damages by overdosing using pesticides. Recently, researchers focused on precision agriculture to make this smart. Sensors are employed to detect leaves of plants on the ground and spray them as much as required. Thus, pesticide dose will be under control. The current paper aims to introduce a wheeled robot that is developed to detect plants by color sensor and spray them. This robot can move between planting rows and detect weeds based on the leave color. A microcontroller-based board was used as the main controller, which sends spray commands to the sprayer nozzle. Outdoor and indoor tests were carried out to study the accuracy of this system. Results of experiments showed that this robot could work with acceptable accuracy in identifying weeds in the field. Thus, this robot can be commercialized for applying in the field to spray pesticides.
\end{abstract}

This is an open-access article under the CC-BY-SA license.

\section{Introduction}

For many years' robotic systems have been widely employed for industrial production and in warehouses. In horticulture and agriculture, research and projects on automatic driverless vehicles and autonomous tractors started in the early 1960s. In recent decades, robotic systems in agriculture have been developed, and researchers were combined new sensor systems, positioning systems (GPS), geographical information systems (GIS), and communication technologies to develop atomized autonomous systems.

Normally, the farmers use pesticides to remove weeds, fungi, or pests by spraying chemicals on the farmyards. By this operation, plant diseases are controlled, and crop yields are improved. A notable issue is using chemical pesticides maybe threaten the environment due to overdose using of pesticides. On the other hand, spraying the pesticide is harmful to the operator [1]. So, it is needed to find a way to control the amount of sprayed chemicals as much as required. Also, it is without human power is 
much desired. Robots are the best solution for this problem because they can work autonomously with acceptable accuracy [2-4]. By this approach, robots can detect weeds on the ground and spray them with pesticides with minimum human supervising for a long time. Consequently, cost reduction environmental and human safety is reached by robotizing this operation [5].

Machine vision tools are used in robots for many applications, such as assembling robots in industry or rescue robots. Color sensors and image processing cameras are developed for this purpose. Also, in precision agriculture, machine vision and image processing are applied vastly. Via this technology, robots can measure moist content, micro and macro elements, fertilizers, and weeds on the soil or subsoil. Thus, geospatial management of inputs will be possible while human labor is reduced [6][7]. Moreover, in the greenhouses, autonomous pesticide robots are interested in researchers because of the cost reduction of this operation [8-12]. In addition to cost reduction, the application of robots in this field helps to keep clean the environment of the greenhouse without human operators [13-15].

Using artificial intelligence in the wheeled mobile robot made it smart for following paths in pesticide spraying with minimum error [16-20]. Also, some other types of robots are employed to spray chemicals on the tree or farmyard. For example, a tree-climbing robot is presented to spread chemicals on leave efficiently [21-24]. Another type of robot which is utilized in spraying pesticides is the drone. They can fly over the crops and spray continually, but control the dose of chemicals based on the Geo-referenced map did not report by this method [25-32].

By reviewing various researches were presented in this area, a combination color sensor with the mobile robot can be proper for pesticide spraying autonomously and make it more effective. In this paper, a test rig robot was developed which can detect weeds between planting rows by color sensor and spraying them automatically. In fact, the research contribution is the design and fabrication of a mobile robot to remove weeds by chemical spraying autonomously.

\section{Method}

\subsection{Structure of Robot}

The proposed system includes some subsystems such as the control unit, color sensor, ultrasonic sensor, trolley module, and spray module. An ultrasonic sensor was used for measuring the distance to the object and finding the pathway. A TCS 3200 module is utilized as a color detector connected to a Micro-Arduino. This module can identify various colors by RGB filter with non-linearity error typically $0.2 \%$ at $50 \mathrm{kHz}$. A program was developed to identify green colors for detecting weeds on the ground surface. A system control unit is an Arduino module to send spray command to sprayer module and control trajectory based on received signals from the ultrasonic sensor. Fig. 1 illustrates the block diagram of components and their connections in this robot. The TCS 3200 module is packed in a case with Arduino micro, and it is unveiled in Fig. 2.

The trolley module includes two electric motors which can drive four wheels via gearboxes. Thus, proper traction is available for motion on the ground. In addition, it can follow trajectory with steering commands. Ultrasonic module SRF05 can measure $4.5 \mathrm{~m}$ by $2 \mathrm{~mm}$ accuracy. The assembled trolley and ultrasonic module are shown in Fig. 3. A column is mounted on the trolley chassis to use as a color detector stand (Fig. 4).

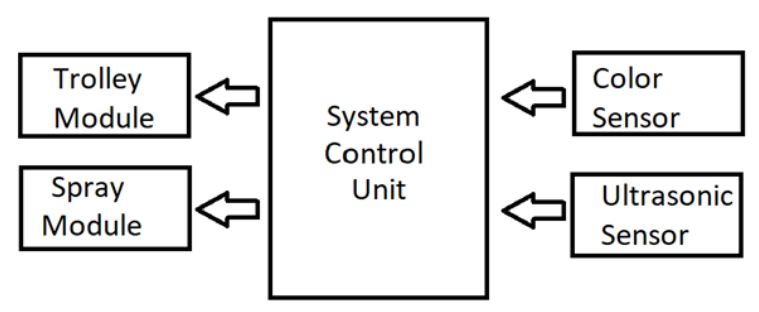

Fig. 1. Block diagram of the system 

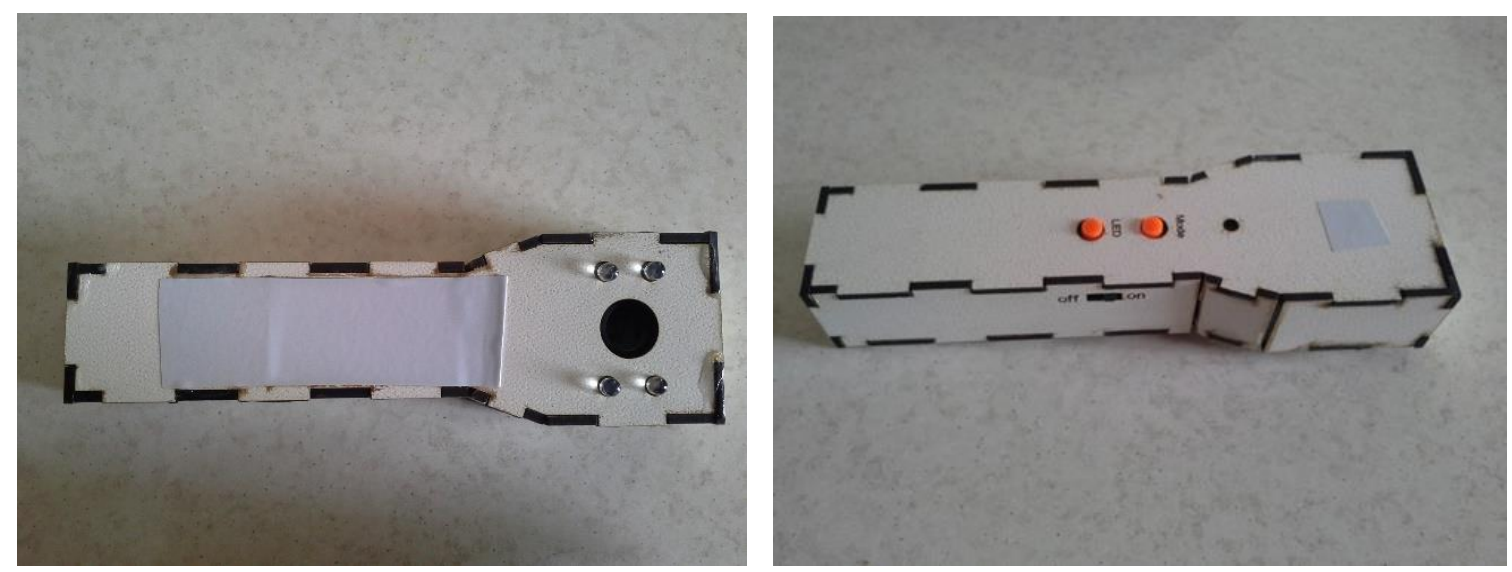

Fig. 2. Color detector and its case

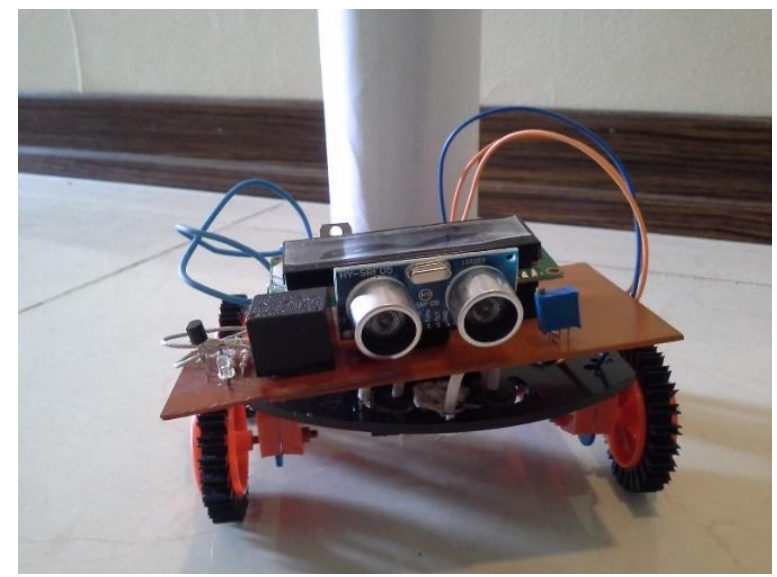

Fig. 3. Ultrasonic module and trolley

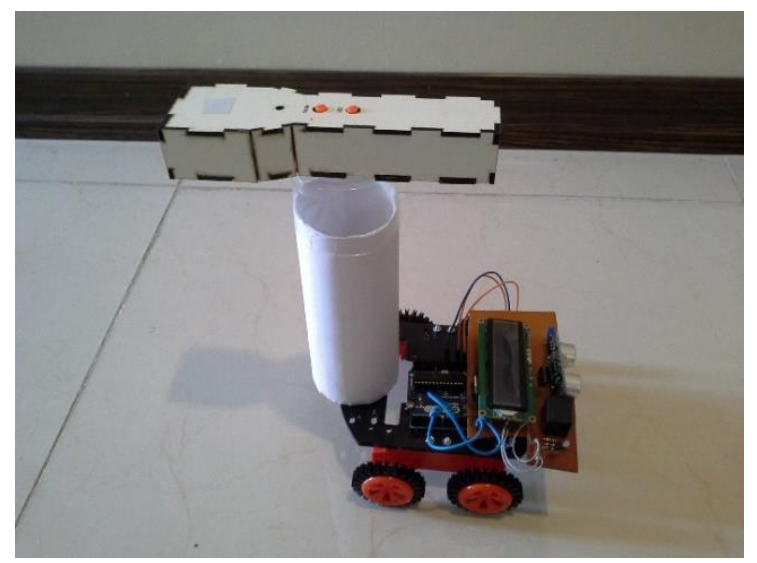

Fig. 4. Color detector mounted on the trolley

The spray module includes a nozzle, solenoid valve, chemicals tank, and connection pipe. The chemical tank is charged by air pressure. The solenoid valve can be on and off by an electrical signal from Arduino. Therefore, it can spray chemicals on the weeds when a color detector identifies weeds on the ground. Kinematic and dynamic models of a robot are necessary for control of that. Consequently, in the following, these two models will be described.

\subsection{Kinematics Model}

In kinematics modeling of the wheeled robot [33][34], for explanations, three assumptions had been considered in robot: 
1- All of the robot parts, particularly wheels, are solids.

2- Robot travels on a flat, smooth, and non-deformable surface.

3- Wheels have rolling motion without skidding or slippage

The mobile robot location on the field is exemplified in Fig. 5. The rotation angle of wheels, $\varphi(t)$, produces movement and heading (direction of motion) in the robot body. The robot position, which is shown by $(x, y, \theta)$, is resting on $\varphi(t)$. The rotation angle of the right wheel and left wheel are denoted by $\varphi 1, \varphi 2$, respectively. The robot linear velocity can be reached by:

$$
v=R\left(\omega_{1}+\omega_{2}\right) / 2
$$

Which, $\omega_{1}$ and $\omega_{2}$ are the angular velocity of wheels, respectively. Furthermore, $R$ is the radius of the wheel. The robot heading and center position can be found by the following equations:

$$
\begin{gathered}
\dot{x}=\left(R \cos \theta\left(\omega_{1}+\omega_{2}\right)\right) / 2 \\
\dot{y}=\left(R \sin \theta\left(\omega_{1}+\omega_{2}\right)\right) / 2 \\
\dot{\theta}=R\left(\omega_{1}-\omega_{2}\right) / 2 L
\end{gathered}
$$

And the velocity of the robot can be calculated by:

$$
\begin{aligned}
& \dot{x}=v \cos \theta \\
& \dot{y}=v \sin \theta
\end{aligned}
$$

Hence, the general matrix for location and heading is as subsequent:

$$
\left[\begin{array}{c}
\dot{x} \\
\dot{y} \\
\dot{\theta}
\end{array}\right]=\left[\begin{array}{cc}
\cos \theta & 0 \\
\sin \theta & 0 \\
0 & 1
\end{array}\right]\left[\begin{array}{l}
v \\
\theta
\end{array}\right]
$$

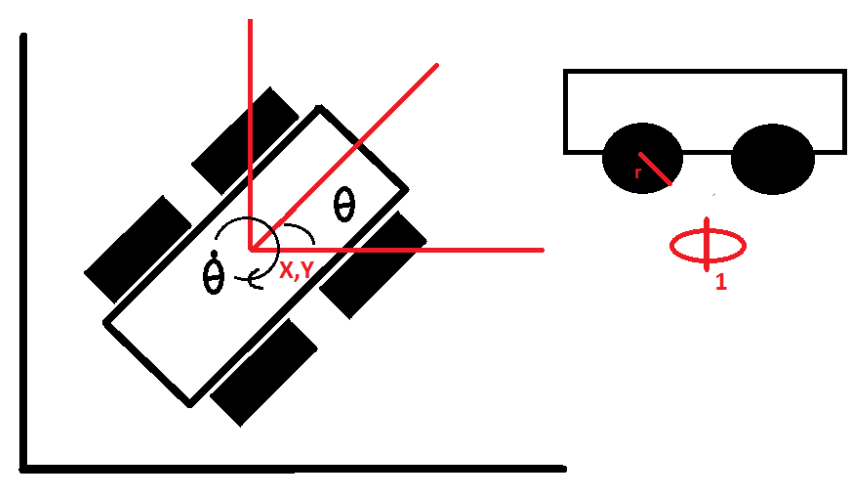

Fig. 5. Location and heading of robot in movement space

Lastly, the kinematics model of the robot is attained as:

$$
\left[\begin{array}{l}
v \\
\dot{\theta}
\end{array}\right]=\left[\begin{array}{cc}
R / 2 & R / 2 \\
R / 2 L & -R / 2 L
\end{array}\right]\left[\begin{array}{c}
\omega_{r} \\
\omega_{l}
\end{array}\right]
$$

\subsection{Dynamics Model}

Commonly, in the dynamics model, the required torque for moving to the desired position will be calculated. Fig. 6 demonstrates the free body diagram of the robot wheel, which is exposed to moment 
on the robot center because of wheels rotation. By solving the dynamic equilibrium conditions, the applied torque will be attained as bellow:

$$
\begin{aligned}
& \ddot{\varphi}_{1}=\frac{\tau}{(M+3 m) r^{2}}+\frac{l^{2}}{l_{r}} \tau_{1}+\frac{\tau}{(M+3 m) r^{2}}+\frac{l^{2}}{l_{r}} \tau_{2} \\
& \ddot{\varphi}_{2}=\frac{\tau}{(M+3 m) r^{2}}+\frac{l^{2}}{l_{r}} \tau_{1}+\frac{\tau}{(M+3 m) r^{2}}+\frac{l^{2}}{l_{r}} \tau_{2}
\end{aligned}
$$

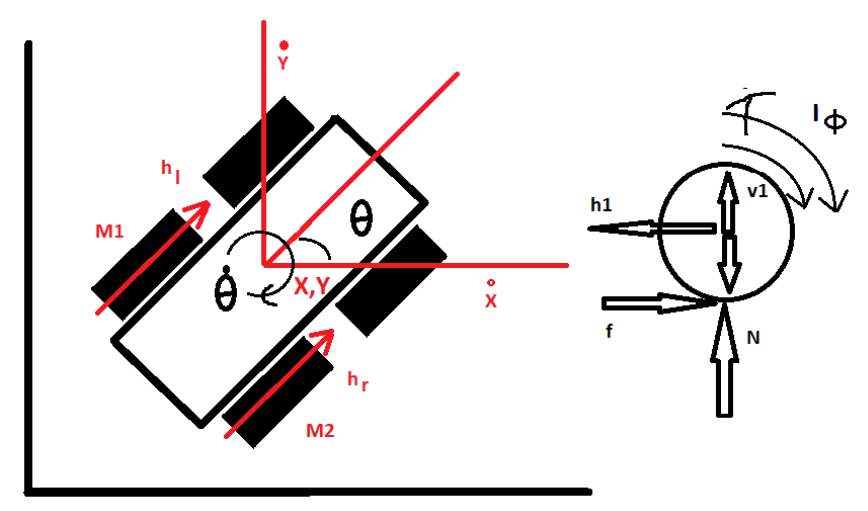

Fig. 6. Free body diagram of a wheeled robot

Thus, the control board produces proper voltage for electrical motors on wheels to follow the planned trajectory. To evaluation of the robot performance, two tests were conducted. The first test is the measurement accuracy of the color detector to find green objects on the ground, which is called an indoor test. The second test, called outdoor, is the evaluation of performance in the field.

In the indoor test, green papers with various areas are installed on the white background randomly, as illustrated in Fig. 7. The area of green papers is between $0.5 \mathrm{~cm} 2$ to $10 \mathrm{~cm} 2$. The ability of the robot to detect green targets was checked. Also, this experiment repeated the height of the detector from $4 \mathrm{~cm}$ to $22 \mathrm{~cm}$. Actually, the relationship between the height of the detector and the area of the green target is studied.

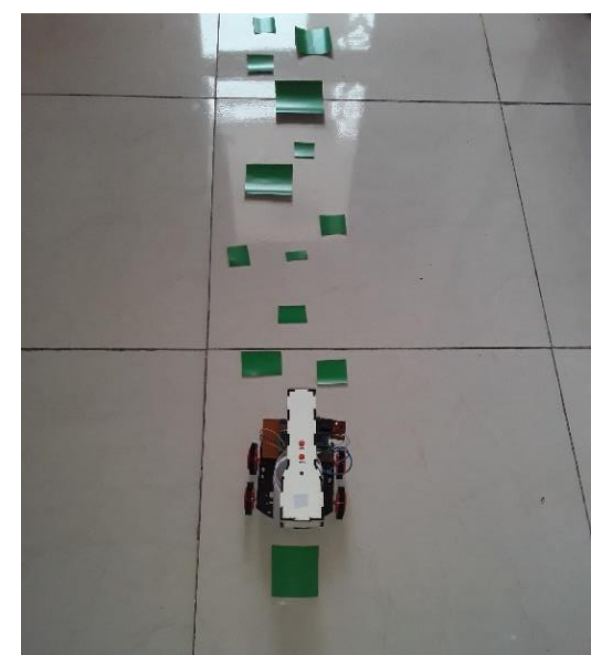

Fig. 7. Indoor test for the evaluation of robot

In the outdoor test, the robot was moved between plant rows. The identified weeds were accounted for, and the area of leaves was measured. Same as the indoor test, the percentage of detected weeds were accounted for, and the relationship between the area and height of the detector was studied. The outdoor test is depicted in Fig. 8. 


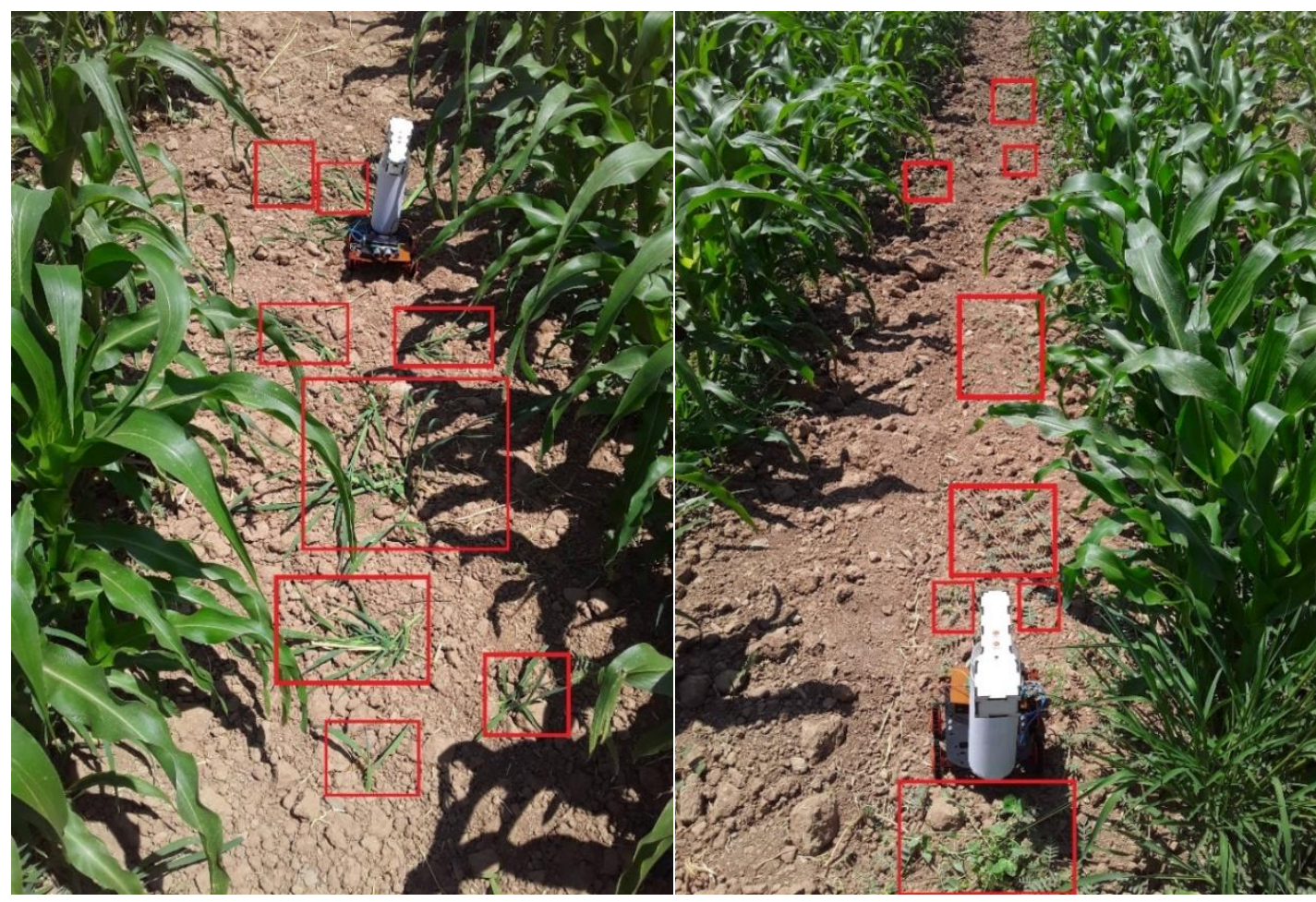

Fig. 8. Indoor test for the evaluation of robot

\section{Results and Discussion}

The results of the indoor test showed that the robot could identify green papers 16 numbers from 20 green papers. Thus, its ability to detect green targets is over 80 percent. Also, the minimum area can be detected by this system is $9 \mathrm{~cm} 2$. Fig. 9 illustrates the relationship between the height of the detector and the area of green paper. As expected, there is a linear relationship between area and height. In other words, by increasing the area of the green target, detecting will be easier.

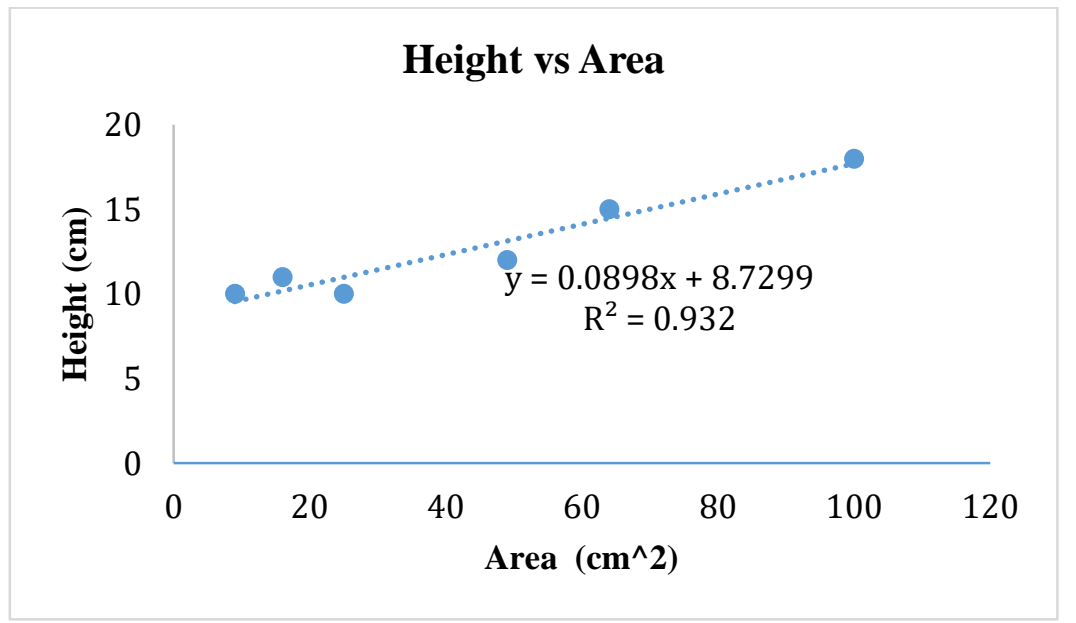

Fig. 9. Relationship between the height of the detector and the area of green paper

Through the outdoor test, the minimum area of weeds identified by the robot is $12 \mathrm{~cm} 2$. Moreover, 72 percent of weeds were detected via the robot. Thus, in real conditions, the performance of the robot in identifying green weeds decreased. Perhaps it is due to differences of light and contrast between soil color and weed color. 


\section{Conclusion}

To decrease the negative overdose of chemical spreading on the agriculture crops, a wheeled robot was equipped to detect a color system to identify weeds between crop rows in the farmyard and spray them. The trajectory of the proposed robot was controlled by ultrasonic radar based on kinematic and dynamic models. The results of indoor and outdoor tests show that the accuracy of the robot in detecting green weeds is acceptable though it decreased in the outdoor test. Thus, this proposed sprayer robot can be developed and employed in the farm for pesticide operation. In future work, this robot will be implemented by a GPS system for more accurate navigation.

Author Contribution: All authors contributed equally to the main contributor to this paper. All authors read and approved the final paper.

Funding: This research received no external funding.

Conflicts of Interest: The authors declare no conflict of interest.

\section{References}

[1] Z. Zhang, and Y. Liu, "Effects of chemical pesticides on human health," Shanghai Journal of Preventive Medicine, vol. 15, no. 8, pp 383-384, 2013.

[2] R. Berenstein, O. B. Shahar, A. Shapiro, and Y. Edan, "Grape clusters and foliage detection algorithms for autonomous selective vineyard sprayer," Intelligent Service Robotics, vol. 3, no. 4, pp. 233-243, October 2010, https://doi.org/10.1007/s11370-010-0078-z.

[3] R. Gadow, A. Candel, and M. Floristán, "Optimized robot trajectory generation for thermal spraying operations and high-quality coatings on free-form surfaces," Surface Coatings Technology, vol. 205, no.4, pp. 1074-1079, 2010, https://doi.org/10.1016/j.surfcoat.2010.08.121.

[4] S. H. Deng, Z. Cai, D. D. Fang, H. Liao, and G. Montavon, “Application of robot offline programming in thermal spraying original research article," Surface Coatings Technology, vol. 206, no. 19, pp. 38753882, 2012, https://doi.org/10.1016/j.surfcoat.2012.03.038.

[5] G. Gao, H. Zhou, and X. Niu, "An intelligent variable spraying decision-making system based on fuzzy neural network for greenhouse mobile robot," Intelligent Computing for Sustainable Energy and Environment, vol. 355, pp. 257-265, 2013, https://doi.org/10.1007/978-3-642-37105-9_29.

[6] J. Peng, Q. He, and Q. Wei, “Design of smart things robot system,” Information Technology Journal, vol. 12, no. 24, pp. 8514-8520, 2013, https://doi.org/10.3923/itj.2013.8514.8520.

[7] J. Peng, X. Li, and Z. Qin, "Swarm-robot system based on wireless sensor network," Journal of Digital Information Management, vol. 10, no.5, pp. 277-283, 2012, https://www.dline.info/fpaper/jdim/v10i5/1.pdf.

[8] P. J. Sammons, T. Furukawa, and A. Bulgin, "Autonomous pesticide spraying robot for use in a greenhouse," Australian Conference on Robotics and Automation, vol. 1, no. 9, 2005. http://www.cse.unsw.edu.au/ acra2005/proceedings/papers/sammons.pdf

[9] N. Nakao, H. Suzuki, T., Kitajima, A. Kuwahara, and T. Yasuno, "Path planning and traveling control for pesticide-spraying robot in greenhouse," Journal of Signal Processing, vol. 21, no. 4, pp.175-178, 2017, https://doi.org/10.2299/jsp.21.175.

[10] V. J. Rincón, M. Grella, P. Marucco, L. E. Alcatrão, J. Sanchez-Hermosilla, and P. Balsari, "Spray performance assessment of a remote-controlled vehicle prototype for pesticide application in greenhouse tomato crops," Science of the Total Environment, vol. 726, p. 138509, 2020, https://doi.org/10.1016/j.scitotenv.2020.138509.

[11] R. González, F. Rodríguez, J. Sánchez-Hermosilla, and J. G. Donaire, "Navigation techniques for mobile robots in greenhouses," Applied Engineering in Agriculture, vol. 25, no. 2, pp. 153-165, 2009, https://doi.org/10.13031/2013.26324. 
[12] U. Zangina, S. Buyamin, M. S. Z. Abidin, and M. S. A. Mahmud, "Agricultural rout planning with variable rate pesticide application in a greenhouse environment," Alexandria Engineering Journal, vol. 60, no. 3, pp. 3007-3020, 2021, https://doi.org/10.1016/j.aej.2021.01.010.

[13] V. J. Rincón, F. C. Páez, and J. Sánchez-Hermosilla, "Potential dermal exposure to operators applying pesticide on greenhouse crops using low-cost equipment," Science of The Total Environment, vol. 630, pp. 1181-1187, 2018, https://doi.org/10.1016/j.scitotenv.2018.02.235.

[14] E. Ozgul and U. Celik, "Design and implementation of semi-autonomous anti-pesticide spraying and insect repellent mobile robot for agricultural applications," 5th International Conference on Electrical and Electronic Engineering (ICEEE), 2018, pp. 233-237, https://doi.org/10.1109/ICEEE2.2018.8391337.

[15] V. Komasilovs, E. Stalidzans, V. Osadcuks, and M. Mednis, "Specification development of robotic system for pesticide spraying in greenhouse," 14th International Symposium on Computational Intelligence and Informatics (CINTI), pp. 453-457, 2013, https://doi.org/10.1109/CINTI.2013.6705239.

[16] Y. Sun, S. Zhang, and W. Li, "Guidance lane detection for pesticide spraying robot in cotton fields," Journal-Tsinghua University, vol. 47, no. 2, p. 206, 2007 , http://en.cnki.com.cn/Article_en/CJFDTotal-QHXB200702011.htm.

[17] D. Deshmukh, D. K. Pratihar, A. K. Deb, H. Ray, and N. Bhattacharyya, "Design and Development of Intelligent Pesticide Spraying System for Agricultural Robot," Advances in Intelligent Systems and Computing, pp. 157-170, 2020, https://doi.org/10.1007/978-3-030-73050-5_16.

[18] N. R. Dhumale and P. C. Bhaskar, "Smart Agricultural Robot for Spraying Pesticide with Image Processing based Disease Classification Technique," International Conference on Emerging Smart Computing and Informatics (ESCI), pp. 604-609, 2021, https://doi.org/10.1109/ESCI50559.2021.9396959.

[19] K. R. Vikram, “Agricultural Robot-A pesticide spraying device," International Journal of Future Generation Communication and Networking, vol. 13, no. 1, 150-160, 2021, https://www.researchgate.net/publication/340827655.

[20] N. S. Bargir, A. S. Vadagaonkar, K. M. Kamble, A. R. Patil, and V. Metkari, "Automatic Pesticide Spraying Machine," International Journal of Advance Scientific Research and Engineering Trends, vol. 6, no. 6, 2021, http://www.ijasret.com/VolumeArticles/FullTextPDF/907_20.AUTOMATIC_PESTICIDE_SPRAYIN G_MACHINE.pdf.

[21] A. G. Bhat, "Arecanut Tree-Climbing and Pesticide Spraying Robot using Servo Controlled Nozzle," Global Conference for Advancement in Technology (GCAT), pp. 1-4, 2019, https://doi.org/10.1109/GCAT47503.2019.8978452.

[22] R. G.Wang, H. B. Huang, Y. Li, and J. W. Yuan, "Design and Analysis of a Novel Tree Climbing Robot Mechanism,” 2020, https://doi.org/10.21203/rs.3.rs-55599/v1.

[23] A. N. Ibrahim, O. Y. Pang, W. K. Yap, and A. S. A. Ghani, "Design of Fast Climbing Robot for Tree with Multiple Diverging Branches," RITA, 395-402, Springer, Singapore, Mechanism, 2018, https://doi.org/10.1007/978-981-13-8323-6_33.

[24] X. Yang, M. Yan, Y. Zhou, and R. Liu, "The Dynamic analysis of Planetary roller screw in Tree-climbing robot," Journal of Physics: Conference Series, vol. 2029, no. 1, p. 012060, IOP Publishing, 2021, https://doi.org/10.1088/1742-6596/2029/1/012060.

[25] Z. Yanliang, L. Qi, and Z. Wei, "Design and test of a six-rotor unmanned aerial vehicle (UAV) electrostatic spraying system for crop protection," International Journal of Agricultural and Biological Engineering, vol. 10, no. 6, pp. 68-76, 2017, https://doi.org/10.25165/j.ijabe.20171006.3460.

[26] X. Zhou, J. He, D. Chen, J. Li, C. Jiang, M. Ji, and M. He, "Human-robot skills transfer interface for UAV-based precision pesticide in dynamic environments," Assembly Automation, 2021, https://doi.org/10.1108/AA-11-2020-0161.

[27] Y. Lan and S. Chen, "Current status and trends of plant protection UAV and its spraying technology in China," International Journal of Precision Agricultural Aviation, vol. 1, no. 1, 2018, https://doi.org/10.33440/j.ijpaa.20180101.0002. 
[28] M. N. Kharim, A. Wayayok, A. R. M. Shariff, A. F. Abdullah, and E. M. Husin, "Droplet deposition density of organic liquid fertilizer at low altitude UAV aerial spraying in rice cultivation," Computers and Electronics in Agriculture, vol. 167, p. 105045, 2019, https://doi.org/10.1016/j.compag.2019.105045.

[29] S. Khan, M. Tufail, M. T. Khan, Z. A. Khan, and J. Iqbal, A. Wasim, "Real-time recognition of spraying area for UAV sprayers using a deep learning approach,” Plos one, vol. 16, no. 4, p. e0249436, 2021, https://doi.org/10.1371/journal.pone.0249436.

[30] C.Wang, J. Song, X. He, Z. Wang, S. Wang, and Y. Meng, "Effect of flight parameters on distribution characteristics of pesticide spraying droplets deposition of plant-protection unmanned aerial vehicle," Transactions of the Chinese Society of Agricultural Engineering, vol. 33, no. 23, pp. 109-116, 2017, https://www.ingentaconnect.com/content/tcsae/tcsae/2017/00000033/00000023/art00014.

[31] W. Qin, X. Xue, S. Zhang, W. Gu, and B. Wang, "Droplet deposition and efficiency of fungicides sprayed with small UAV against wheat powdery mildew," International Journal of Agricultural and Biological Engineering, vol. 11, no. 2, pp. 27-32, 2018, https://doi.org/10.25165/j.ijabe.20181102.3157.

[32] Y. Meng, J. Su, J. Song, W. H. Chen, and Y. Lan, "Experimental evaluation of UAV spraying for peach trees of different shapes: Effects of operational parameters on droplet distribution," Computers and Electronics in Agriculture, vol. 170, p. 105282, 2020, https://doi.org/10.1016/j.compag.2020.105282.

[33] M. Gohari, M. Tahmasebi, and A. Nozari, "Application of machine learning for NonHolonomic mobile robot trajectory controlling," 4th International Conference on Computer and Knowledge Engineering (ICCKE), pp. 42-46, 2014, https://doi.org/10.1109/ICCKE.2014.6993354.

[34] M. Gohari, M. Tahmasebi, and M. Sadeghian, "The iterative learning method in NonHolonomic mobile robot," NCMEISO1. 